\title{
openheart Specialist valve clinic in a cardiac centre: 10-year experience
}

\author{
John B Chambers (D) , Denise Parkin, Helen Rimington, Sheila Subbiah, \\ Brian Campbell, Camelia Demetrescu, Anna Hayes, Ronak Rajani
}

\begin{abstract}
- Additional material is published online only. To view please visit the journal online (http://dx.doi.org/10.1136/ openhrt-2020-001262).
\end{abstract}

To cite: Chambers JB, Parkin D, Rimington $\mathrm{H}$, et al. Specialist valve clinic in a cardiac centre: 10-year experience. Open Heart 2020;7:e001262. doi:10.1136/ openhrt-2020-001262

Received 13 February 2020 Revised 12 March 2020 Accepted 16 March 2020
Check for updates

\section{(C) Author(s) (or their} employer(s)) 2020. Re-use permitted under CC BY-NC. No commercial re-use. See rights and permissions. Published by BMJ.

Guy's and St Thomas' Hospital, London, UK

\section{Correspondence to} Dr John B Chambers; jboydchambers@aol.com

\section{ABSTRACT}

Aims Guidelines recommend specialist valve clinics as best practice for the assessment and conservative management of patients with heart valve disease. However, there is little guidance on how to set up and organise a clinic. The aim of this study is to describe a clinic run by a multidisciplinary team consisting of cardiologists, physiologist/scientists and a nurse. Methods The clinical and organisational aims of the clinic, inclusion and exclusion criteria, and links with other services are described. The methods of training nonclinical staff are detailed. Data were prospectively entered onto a database and the study consisted of an analysis of the clinical characteristics and outcomes of all patients seen between 1 January 2009 and 31 December 2018. Results There were 2126 new patients and 9522 visits in the 10-year period. The mean age was 64.8 and $55 \%$ were male. Of the visits, $3587(38 \%)$ were to the cardiologists, $4092(43 \%)$ to the physiologist/scientists and $1843(19 \%)$ to the nurse. The outcomes from the cardiologist clinics were cardiology follow-up in 460 (30\%), referral for surgery in $354(23 \%)$, referral to the physiologist/scientist clinic in $412(27 \%)$ or to the nurse clinic in 65 (4.3\%) and discharge in $230(15 \%)$. The cardiologist needed to see $6 \%$ from the nurse clinic and $10 \%$ from the physiologist/scientist clinic, while advice alone was sufficient in $10 \%$ and $9 \%$.

Conclusion A multidisciplinary specialist valve clinic is feasible and sustainable in the long term.

\section{INTRODUCTION}

National and International guidelines $^{12}$ recommend specialist valve clinics for the outpatient care of patients with heart valve disease. There are numerous potential models $^{3}$ involving cardiologists, surgeons, nurses, physiologists or clinical scientists. Multidisciplinary valve clinics are clinically more effective than general clinics, ${ }^{45}$ are better patient focused $^{6-8}$ and more costeffective. ${ }^{9}$ However, there is little published information on the details of how they are run and this would be useful for clinicians wanting to set up a new valve clinic.

At Guy's and St Thomas' Hospitals (GSTT), a multidisciplinary valve clinic involving cardiologists, nurses and expert physiologists evolved from a research clinic in 2007

\section{Key questions}

What is already known about this subject?

- Specialist valve clinics are seen as best practice. However, little information is available to guide hospitals wanting to set them up.

What does this study add?

- This study describes one model of valve clinic including cardiologists, physiologist/scientists and a nurse. Compliance with guidelines was better than for patients with valve disease seen in a general cardiology clinic.

How might this impact on clinical practice?

- This descriptive study should guide cardiologists, physiologist/scientists or nurses in setting up a clinic safely and effectively.

and data were collected from 2009. The core aim of the clinic was to follow patients with moderate or severe native disease until the optimum time for intervention, to then refer them to the most appropriate surgeon or interventional cardiologist and afterwards to resume long-term care.

We describe the structure of the clinic, the patients seen and outcomes for the 10-year period from 2009 to 2018.

\section{METHODS}

All 9522 clinic visits from 1 January 2009 to 31 December 2018 at GSTT were examined. The hospital provides secondary level care to a local population of 750000 , and tertiary care to approximately 6 million in South East England.

Patients were seen by one or more of a team consisting initially of a cardiologist specialising in valve disease, two expert physiologist/ scientists and a valve specialist nurse running one whole clinic day per week. The nurse and physiologist/scientists all had at least 15 years experience and received specialist training by sitting in the valve clinic with a consultant cardiologist typically for six clinics. Two of the physiologist/scientists attended MSc 
modules in clinical assessment. The cardiologist then tested the ability of the nurse and physiologist/scientists to assess patients by requiring them to complete clinical assessment sheets (online supplementary data) for 20 patients and comparing these with his own assessment. They were only allowed to lead a devolved clinic if all 20 were correct. The cardiologist had a departmental sonographer allocated to perform echocardiograms for all the patients on his list. The cardiologist and nurse appointments were booked every $30 \mathrm{~min}$ giving a capacity of eight cases per session. Many patients were seen before and after their echocardiogram and there were usually an additional two referrals from the nurse or physiologist/ scientist clinics. The physiologist/scientist appointments were booked every 1 hour giving a capacity of four cases per session. Two clinical scientists joined the team in 2017 and a second consultant and second departmental sonographer in 2018. Each week the clinic now occupies three consultant cardiologist sessions, four physiologist/ scientist sessions and two nurse sessions.

\section{Inclusions and exclusions}

Patients were included with: moderate or severe native disease; repaired or replaced valves; treated infective endocarditis; dilated aortas or the risk of developing aortic dilatation. Referrals for patients with frailty or multiple morbidities were forwarded to the elderly care unit or, with inoperable secondary mitral regurgitation, to the heart failure service. Patients becoming infirm or otherwise no longer suitable for surgery could have been referred to these departments but in practice tended to be kept in the valve clinic by patient choice. The clinic has links with cardiac specialists (eg, electrophysiology) and non-cardiac specialist services (eg, dentistry).

\section{Roles of each discipline}

All disciplines conducted a symptom enquiry, a physical examination and a review of medication. All offered life style advice about smoking cessation, weight control and dental surveillance. Emails and telephone follow-ups were conducted by the nurse or cardiologists. All disciplines recorded the consultation electronically and communicated with the general practitioner and referring physician.

The cardiologists saw new patients not already known to GSTT, complex patients with multiple comorbidities and those close to surgery. They also saw or discussed patients referred from the physiologist/scientist and nurse-led clinics according to clinical or echocardiographic thresholds on an agreed protocol (see online supplementary information).

The physiologist/scientist monitored patients with stable moderate or severe native disease or with replaced or repaired valves requiring routine echocardiography. The nurse monitored patients after surgery who did not require echocardiography and also saw some patients who had made direct helpline or email contact. She also coordinated the clinic, managing appointments,

\section{Roles of the GSTT valve clinic}

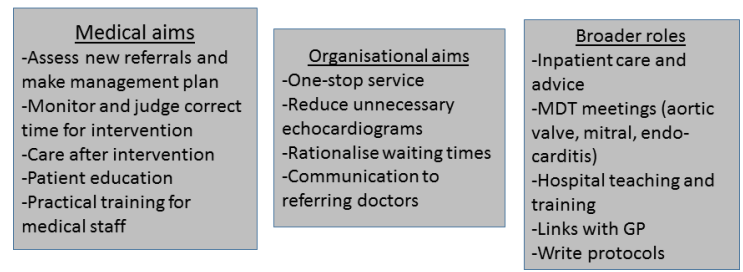

Figure 1 Clinical and organisation aims of a specialist valve clinic. GP, general practitioner; GSTT, Guy's and St Thomas' Hospitals; MDT, multidisciplinary team.

maintained the audit database, followed up results and ran the telephone and email helplines. The helpline was not formally assessed. However, approximately 50 contacts per week occur of which about one half are for changing appointments, and about half about medication, insurance, MR compatibility, anticoagulation bridging or new symptoms.

\section{Organisation}

The medical and organisational aims are given in figure 1 . A meeting was held every week before the main clinic day to discuss results or outcomes and to triage new referrals (to the cardiologist, physiologist/scientist or nurse clinics with the criteria given under roles of each discipline). Cases were discussed with cardiac surgeons as necessary at a weekly multidisciplinary meeting or referred to separate transcatheter aortic valve implantation (TAVI), or transcatheter mitral or paraprosthetic closure teams. The TAVI service has a separate outpatient clinic run by two cardiologists, one surgeon and one clinical scientist. GSTT also has weekly multidisciplinary team (MDT) for endocarditis, mitral surgery and aortic pathology. There are three mitral repair surgeons, six surgeons performing aortic valve replacements and three specialising in aortic surgery. There are two interventional cardiologists performing TAVI or other transcatheter procedures and a third performing transcatheter repairs of paraprosthetic regurgitation.

\section{Investigations}

All patients needing echocardiography had this at the time of the clinical visit (unless this had been performed prior to the referral). There was an exercise physiologist available for one-stop treadmill exercise tests, a pacing physiologist for pacemaker checks, clinic nurses for blood tests (including B-type natriuretic peptide levels for patients unable to exercise or who had symptoms with more than one possible cause). All new cases had a 12-lead ECG. Stress echocardiography, CT and CMR (cardiac magnetic resonance) required booking. The frequency of follow-up and echocardiography was guided by international guidelines ${ }^{10}$ (online supplementary information). 


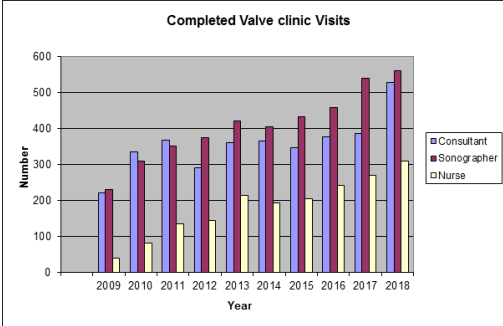

Figure 2 Number of patients seen by each discipline.

\section{Analysis}

Specifically designed forms (see online supplementary information) were completed for each clinic visit and data entered onto a trust approved database. Mean values were calculated for age. No statistical analysis was appropriate for this descriptive study.

Possible outcomes after each visit were: (1) ongoing surveillance in the same clinic or a different clinic arm; (2) referral for surgery/intervention; (3) discussion or internal referral to the cardiologist from the physiologist/scientist or nurse-led clinics and (4) discharge.

\section{RESULTS}

Over 10 years, there were 9522 clinic visits and 2126 new patients (figure 2), 3587 (38\%) to see a cardiologist, 4092 $(43 \%)$ a physiologist/scientist and $1843(19 \%)$ the nurse. The mean age of the patients was 64.8 (range 17-99); $1173(55 \%)$ were male and $953(45 \%)$ were female. The rate of failure to attend was $7.7 \%$ (compared with the cardiac department average of $13.0 \%$ ).

Of the 2126 new patients, 1459 (68.6\%) had native valve disease, 676 severe, 589 moderate and 194 mild (table 1 ). There were $575(27 \%)$ with repaired or replaced valves (table 2). A further 36 (1.7\%) had aortic dilatation and $56(2.6 \%)$ had miscellaneous problems (VSD (ventricular septal defect), fibroelastomas, atrial thrombus, pulmonary valve disease).
Table 2 Nature of 561 new patients with operated valve disease (\% of 561 )

\begin{tabular}{lcll} 
& Normal & Abnormal & Total \\
\hline Aortic valve replacement & $281(50.1)$ & $40(7.1)$ & $321(57.2)$ \\
$\begin{array}{l}\text { Mitral valve replacement } \\
71(12.7)\end{array}$ & $10(1.8)$ & $81(14.4)$ \\
$\begin{array}{l}\text { Mitral valve repair } \\
\text { Dual aortic and mitral } \\
\text { replacement }\end{array}$ & $30(5.3)$ & $12(2.0)$ & $42(7.5)$ \\
$\begin{array}{l}\text { Isolated tricuspid } \\
\text { replacement/repair }\end{array}$ & $11(2.0)$ & 0 & $11(2.0)$ \\
Ross & $17(3.0)$ & $7(1.2)$ & $24(4.3)$ \\
\hline
\end{tabular}

\section{Cardiologist clinic}

Of the 2126 new patients, 605 were triaged directly to the nurse or clinical scientist clinics. Therefore, the cardiologists saw 1521 new cases, 426 (28\%) referred from other cardiologists and 243 (16\%) from physicians at GSTT, 475 (31\%) from cardiologists outside the trust, 204 (13\%) from open access echocardiography or murmur clinics and $76(5 \%)$ following inpatient treatment for infective endocarditis. There was missing information for $97(6 \%)$. The outcome from the initial visits is shown in figure 3 . Discharges were because the valve disease was too mild for follow-up, the patients asked for local follow-up requested or the patient was not suitable for surgery.

There were 2066 follow-up visits. The number tended to increase latterly as physiologist/ scientist spaces reduced. Exercise tests were performed at $430(12 \%)$ of the 3587 new and follow-up visits.

\section{Physiologist/scientist clinic}

There were 4092 (43\% of clinic total) visits. A review from a cardiologist at the clinic was required in 407 (9.9\%). These included 85 arrhythmias, and 67 patients who were subsequently referred for surgery or transcatheter intervention. Other reasons included attaining protocol

Table 1 Abnormalities in 1459 new patients with native valve disease given as number (\% of 1459)

\begin{tabular}{|c|c|c|c|c|}
\hline & Stenosis & Regurg & Mixed & Total \\
\hline \multicolumn{5}{|c|}{ Moderate and severe $(n=1265)$} \\
\hline Aortic & $478(32.8)$ & $155(10.6)$ & $19(1.3)$ & $652(44.7)$ \\
\hline Tricuspid & 0 & $61(4.2)$ & 0 & $61(4.2)$ \\
\hline Multivalve & & & $39(2.7)$ & $39(2.7)$ \\
\hline Mitral prolapse & & $18(1.2)$ & & $18(1.2)$ \\
\hline Bicuspid aortic valve & & & & $34(2.3)$ \\
\hline Postendocarditis & & $13(0.9)$ & & $13(0.9)$ \\
\hline Rheumatic valve disease & & & $20(1.4)$ & $20(1.4)$ \\
\hline Aortic sclerosis & & & & $59(4.0)$ \\
\hline
\end{tabular}




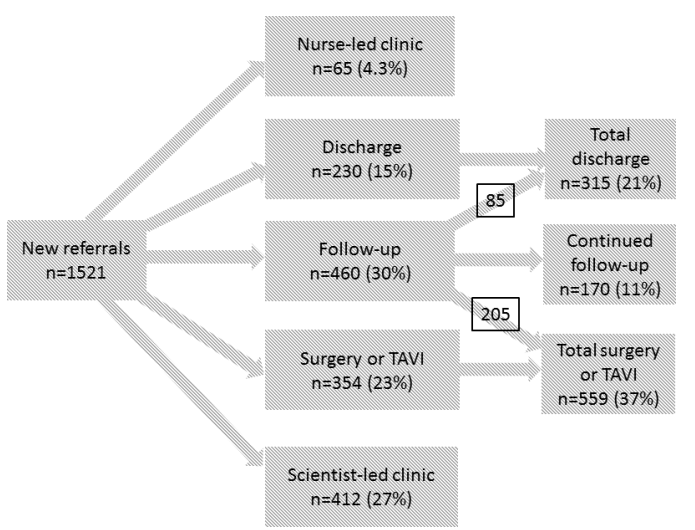

Figure 3 Numbers and outcome of new patients seen in the cardiologist clinic over a 10-year period. TAVI, Transarterial valve implantation.

echocardiographic thresholds (see online supplementary material) not requiring surgery, the development of new symptoms from a non-valve disease process or a clinical event, questions about medication, or at the patient's request. Our protocols trigger discussion with the cardiologist at a relatively low threshold for safety so surgery is not necessarily indicated. Advice alone was needed in 386 $(9.4 \%)$. There was a slight increase in requests for review or advice when a new physiologist/scientist started. Exercise tests were requested in 115 (2.8\%) for symptom evaluation or routine monitoring. Discharges from the clinic were few and so not quantified here. The main reasons were a patient requesting local follow-up, or failing health making repeat visits futile.

\section{Nurse clinic}

There were 1843 visits, $(9.4 \%$ of the clinic total). A cardiologist review was required in $115(6.2 \%)$ including 84 $(4.6 \%)$ with a new or problematic arrhythmias and three patients requiring redo surgery. Other reasons included symptoms or events, medication queries or patient request. Advice alone was needed for 192 (10.4\%).

Exercise tests were requested at 40 (2.2\% of visits) and unscheduled echocardiograms were needed on 78 $(9.7 \%)$ visits, mostly for absent post operative baseline studies but this reduced with improved surgical discharge management. Discharges from clinic were as for the clinical scientist-led clinic but were few.

\section{Dental}

Data collection for dental advice and status changed in 2013 and are given from this date. At the physiologist/ scientist clinic, $3315(81.0 \%)$ visits recorded that dental surveillance occurred, while at $393(9.6 \%)$ visits the patient required a reminder or chose not to attend. At the nurse clinic the numbers were $1495(81.1 \%)$ and $173(9.4 \%)$, respectively. The remaining patients were edentulous. This information was not collected from the consultant clinics. Solutions to dental access were offered (Surgical Clinical Decision Unit, Phobia Clinics, Free Student Clinics) if needed to improve compliance.

\section{DISCUSSION}

This is the first detailed description of a multidisciplinary specialist valve clinic with 9522 visits in 2126 patients over a 10-year period.

The key to a valve clinic is having all disciplines declaring an interest and developing and maintaining specialist competencies ${ }^{11}$ in valve disease. This improves adherence to guidelines concerning investigation, frequency of follow-up and intervention. ${ }^{5}$ Symptoms are detected earlier in aortic stenosis (AS). ${ }^{4}$ In an unpublished audit, we showed that $10 \%$ of patients referred to our clinic as asymptomatic did in fact have symptoms.

The organisational aims (figure 1) were met in that onestop echocardiography was the rule. This was only available in $82 \%$ of valve clinics in a survey conducted by the British Heart Valve Society in 2015. ${ }^{3}$ Exercise tests are rarely performed in non-specialist clinics in the whole of Europe ${ }^{12}$ despite being recommended in international guidance. ${ }^{10}$ Our rate of testing, $12 \%$, also appeared low as a percentage of total visits. However, many patients already had clear symptoms on the history or had multiple comorbidities. Exercise tests were performed no more frequently than annually while clinic visits for severe valve disease occurred 6 monthly. In all, 797 tests were performed for asymptomatic AS and symptoms were revealed in $38 \%$ of patients with apparently asymptomatic severe AS. ${ }^{13}$

We performed an unpublished audit of all 134 patients with valve disease seen over an 18-month year period in a general cardiology clinic compared with 173 patients in the specialist valve clinic. Concordance with management guidelines was better in the specialist valve clinic $(97 \%$ vs $62 \% ; \mathrm{p}<0.001$ ) with lower rates of inappropriate frequency of echocardiography ( $1.6 \%$ vs $85 \%$; $\mathrm{p}<0.001)$. Advice about dental surveillance and antibiotic prophylaxis was recorded in every case in the valve clinic and in no case in the general cardiology clinic. Others have shown ${ }^{5}$ that physiologist/scientist-led clinics improve adherence to guidelines compared with cardiologist-led clinics.

The reduction in unnecessary echocardiograms and lower salary costs mean that a devolved clinic saves about $€ 5000$ for every 100 patients seen in either a physiologist/scientist-led or nurse-led clinic. ${ }^{9}$

The core specialist is usually a non-invasive cardiologist with coexistent specialisation in cardiac imaging or heart failure. However, in the 2015 British Heart Valve Society survey $^{3}$ an interventional cardiologist led in $4(12 \%)$ and a surgeon in $2(6 \%)$ clinics. Cardiac centres like ours are expected to have clinics for general valve cases and others for patients referred for consideration of surgery or transcatheter procedures. It is important that a centre does not develop only interventional clinics because this fails to address the needs of the majority of patients who require 'watchful waiting'. 14

In the EuroHeart Survey ${ }^{12}$ more than half of patients with valve disease were seen in general medical clinics. By 2015 , a survey conducted in the $\mathrm{UK}^{3}$ still showed that only $11 \%$ of district hospitals and $60 \%$ of cardiac centres had 
valve clinics. Physiologist/scientist-led clinics alone were offered in $8(24 \%)$ and combined with cardiologists in $4(12 \%)$. Nurse-led clinics alone were offered in $3(9 \%)$ and combined clinics in $4(12 \%)$. It is important to have a cardiologist clinic running at the same time as the nurse and physiologist/scientist clinics to offer advice which was needed for $10 \%$ of patients and a clinical review for a further $10 \%$ from the scientist-led clinic.

There is little similar work. The EuroHeart Survey ${ }^{12}$ gathered data on patients with valve disease being seen in cardiac medical and surgical outpatients for follow-up but also as inpatients. As in our clinic, AS and mitral regurgitation dominated with 34\% having AS compared with our $38 \%$ and $25 \%$ having mitral regurgitation compared with our $36 \%$. We found many fewer with multiple valve disease, $20 \%$ in EuroHeart Survey compared with 3\% in ours. This is likely a methodological difference since we only classified valve lesions greater than mild in grade.

Some centres discharge patients after surgery and ask them to present if problems occur. ${ }^{15}$ Unfortunately, patients do not necessarily recognise problems and frequently need advice about anticoagulation control, lifestyle modification and dental surveillance ${ }^{8}$ as confirmed in the results in our current study. Without a valve clinic, follow-up is otherwise patchy. ${ }^{716}$

The number of valve clinics is expected to rise as national and international recommendations on best practice are implemented. It is vital that these are part of a network of care seamlessly linking the community, district hospitals and cardiac centres. This will place further demands on an already stretched system although methods of reducing this will emerge. For example, a murmur clinic ${ }^{17}$ has been shown to reduce the need for standard echocardiograms and to allow follow-up of selected patients in the community. Valve clinics place a particular strain on the echocardiography workforce but are also a way of demonstrating equivalence with the new career structure and are likely to become an established part of the training of clinical scientists.

\section{LIMITATIONS}

Staffing the valve clinic with an expert physiologist or clinical scientist inevitably reduces the work-force for general work. It is also essential that training is offered to ensure that these physiologist/scientists have the requisite clinical skills. It would not be safe for the valve clinic to be part of the general roster. When the physiologist/scientist-led clinics were introduced 10 years ago, they were staffed by a senior cardiac physiologist with specialist training in valve disease. With current training and workforce structures, this has evolved into a role for clinical scientists allowing additional protection for patients through statutory regulation.

It may be difficult to arrange one-stop exercise tests although we accomplished this by having a cardiac physiologist on stand-by while performing tape analyses.
In conclusion, a multidisciplinary specialist valve clinic is feasible and sustainable in the long term. It ensures that patients see members of a MDT with appropriate competencies and delivers its clinical and organisational aims successfully.

Contributors JBC, DP and HR founded the cardiology, nurse and physiologist components of the valve clinic. DP collected and analysed the data and JBC wrote the first draft of this article. RR joined the cardiology-led clinic and all the other coauthors ran physiologist/scientist-led clinics. All coauthors contributed to the writing of subsequent drafts and the final version of the paper.

Funding The authors have not declared a specific grant for this research from any funding agency in the public, commercial or not-for-profit sectors.

Competing interests None declared.

Patient consent for publication Not required.

Ethics approval The study was managed and conducted in accordance with the Declaration of Helsinki, and latest Good Clinical Practice (GCP) guidelines. It received Trust approval from the audit committee (project 9787).

Provenance and peer review Not commissioned; externally peer reviewed.

Data availability statement Data are available on reasonable request.

Open access This is an open access article distributed in accordance with the Creative Commons Attribution Non Commercial (CC BY-NC 4.0) license, which permits others to distribute, remix, adapt, build upon this work non-commercially, and license their derivative works on different terms, provided the original work is properly cited, appropriate credit is given, any changes made indicated, and the use is non-commercial. See: http://creativecommons.org/licenses/by-nc/4.0/.

ORCID iD

John B Chambers http://orcid.org/0000-0002-7169-3786

\section{REFERENCES}

1 Lancellotti P, Rosenhek R, Pibarot P, et al. Heart valve clinics: organisation, structure and experiences. Eur Heart $J$ 2013;34:1597-606.

2 Chambers JB, Ray S, Prendergast B, et al. Specialist valve clinics: recommendations from the British heart valve Society Working group on improving quality in the delivery of care for patients with heart valve disease. Heart 2013;99:1714-6.

3 Bhattacharyya S, Pavitt C, Lloyd G, et al. Provision, organization and models of heart valve clinics within the United Kingdom. QJM 2015;108:113-7.

4 Zilberszac R, Lancellotti P, Gilon D, et al. Role of a heart valve clinic programme in the management of patients with aortic stenosis. Eur Heart J Cardiovasc Imaging 2017;18:138-44.

5 Taggu W, Topham A, Hart L, et al. A cardiac sonographer led follow up clinic for heart valve disease. Int J Cardiol 2009;132:240-3.

6 Anscombe A, Cadet J. Follow-Up of patients after heart valve surgery: setting up a nurse-led clinic. Br J Cardiac Nursing 2008;3:320-3.

7 Parkin D, Chambers J. Routine follow-up for patients with prosthetic valves: the value of a nurse-led valve clinic. $\mathrm{Br} J \mathrm{Cardiol}$ 2012;19:204-6.

8 McLachlan A, Sutton T, Ding P, et al. A nurse practitioner clinic: a novel approach to supporting patients following heart valve surgery. Heart Lung Circ 2015;24:1126-33.

9 lonescu A, McKenzie C, Chambers JB. Are valve clinics a sound investment for the health service? A cost-effectiveness model and an automated tool for cost estimation. Open Heart 2015;2:e000275.

10 Baumgartner H, Falk V, Bax JJ, et al. 2017 ESC/EACTS guidelines for the management of valvular heart disease. Eur Heart $J$ 2017;38:2739-91.

11 Chambers J, Campbell B, Wilson J, et al. How should specialist competencies in heart valve disease be recognized? QJM 2015;108:353-4.

12 lung B, Baron G, Butchart EG, et al. A prospective survey of patients with valvular heart disease in Europe: the Euro heart survey on valvular heart disease. Eur Heart J 2003;24:1231-43.

13 Saeed S, Rajani R, Seifert R, et al. Exercise testing in patients with asymptomatic moderate or severe aortic stenosis. Heart 2018;104:1836-42. 
14 Chambers JB. Specialist valve clinic: why, who and how? Heart 2019;105:1913-20.

15 Mahy IR, Dougall H, Buckley A, et al. Routine hospital based follow up for patients with mechanical valve prostheses: is it worthwhile? Heart 1999;82:520-2.
16 Alaour B, Menexi C, Shah BN. Clinical and echocardiographic followup of patients following surgical heart valve repair or replacement: a tertiary centre experience. Echo Res Practice 2018;5:113-9.

17 Draper J, Subbiah S, Bailey R, et al. Murmur clinic: validation of a new model for detecting heart valve disease. Heart 2019;105:56-9. 